Methods There are no current standards against which to audit the departments learning curve for adoption of sentinel lymph node mapping as endometrial cancer staging. We identified published quality indicators for sentinel lymph node mapping - including $<5 \%$ false negative rate, $>20$ cases per surgeon performing the procedure, successful bilateral mapping in $>50 \%$ of cases. Our local gynae oncology database was searched to identify all cases of sentinel lymph node dissection for endometrial and cervical cancer. Data from the gynae oncology database and the patients electronic clinical record was then collated and analysed using excel.

Results 43 patients were identified having undergone a sentinel lymph node biopsy \pm lymphadenectomy for endometrial or cervical cancer. Bilateral sentinel lymph nodes were mapped in $67.4 \%$ of cases. In the first $21 / 43$ patients $57.1 \%$ were mapped, comparative to $77.3 \%$ in latter $22 / 43$ patients. 38 sides with successful lymph node mapping and lymphadenectomy were identified. Sentinel lymph nodes had a 33\% sensitivity for identifying lymph node metastasis in the first half of the data set comparative to $100 \%$ in the latter half.

Conclusions The data demonstrated a significant learning curve, within the department, in the successful mapping of sentinel lymph nodes in endometrial cancer.

\section{EPV143/\#656 RETROSPECTIVE DATA ANALYSIS OF HOSPITAL SANTA MARCELINA, SAO PAULO-SP, BRAZIL}

M Mesquita*, M Brandão, C Sousa, J Barbosa, S Sanches, I Manchini, C Gomez, M Silva, T Almeida. Casa de Saúde Santa Marcelina, Gynecologic Oncology, Sao Paulo, Brazil

\subsection{6/ijgc-2021-IGCS.213}

Objectives Evaluate the epidemiological aspects of patients with endometrial cancer, based on statistics from the Oncology Gynecology Center of Santa Marcelina Hospital in Sao Paulo, Brazil between 2011 to 2018 .

Methods Evaluate the epidemiological aspects of patients with endometrial cancer (EC), based on statistics from the Oncology Gynecology Center of Santa Marcelina Hospital in Sao Paulo, Brazil between 2011 to 2018 .

Results The median age at diagnosis was 63 years and the diagnosed cases were predominantly white ethnicity (51\%). Bleeding after menopause was the most frequent symptom reported (77.8\%). Among the cases analyzed, 36 nulliparous patients presented endometrial cancer (15\%). The most prevalent histological type was endometrioid adenocarcinoma $(66.1 \%)$. The most frequent tumor staging was IA with $30.9 \%$, followed by IB $18.83 \%$, II $2 \%$, IIIA $8 \%$, IIIB $9.2 \%$ IIIC1 $4.6 \%$, IIIC2 $6.69 \%$, IVA $0.42 \%$ and IVB $16.74 \%$. Surgical staging with hysterectomy and bilateral adnexectomy represented $76.9 \%$ and the most frequent adjuvant treatment was brachytherapy (53.1\%). Seventy patients underwent brachytherapy and pelvic radiotherapy (29.9\%) and 38 patients underwent adjuvant brachytherapy, radiotherapy and chemotherapy as an adjuvant (15.9\%). An overall survival rate of $65 \%$ and a mortality rate of $29 \%$ over the 5 -year period have been identified.

Conclusions EC is the eighth most frequent gynecological tumor in Brazil. Data analysis allowed to corroborate the most common clinical symptom and the frequent histological type in the literature. This neoplasm classically presents early symptoms and curative treatment, however the data analysis shows a high death rate and diagnosis of advanced disease.
So, the endometrioid type, doesn't have the best prognosis always and needs a better molecular analysis to optimize therapy,to reduce mortality.

\section{EPV144/\#76 THE TUNISIAN COUNTRY-SPECIFIC GUIDELINES FOR ENDOMETRIAL CANCER}

${ }^{1} \mathrm{M}$ Ghalleb*, 'L Naija, ${ }^{1} \mathrm{~A}$ Chabchoub, ${ }^{1} \mathrm{I}$ Zemni, ${ }^{2} \mathrm{~S}$ Zaraa, ${ }^{2} \mathrm{~S}$ Yahyaoui, ${ }^{3} \mathrm{~F}$ Mghirbi, ${ }^{3} \mathrm{M}$ Ayadi, ${ }^{3} \mathrm{~N}$ Chraiet, ${ }^{1} \mathrm{M}$ Slimane, ${ }^{4} \mathrm{~L}$ Charfi, ${ }^{4} \mathrm{~K}$ Mrad, ${ }^{2} \mathrm{C}$ Nasr, ${ }^{3} \mathrm{~A}$ Mezlini, ${ }^{1} \mathrm{M}$ Hechiche, ${ }^{1} \mathrm{~K}$ Rahal. 'Salah Azaiez Institute of Oncology, Surgical Oncology, Tunis, Tunisia; ${ }^{2}$ Salah Azaiez Institute of Oncology, Radiation Oncology, Tunis, Tunisia; ${ }^{3}$ Salah Azaiez Institute of Oncology, Medical Oncology, Tunis, Tunisia; ${ }^{4}$ Salah Azaiez Institute of Oncology, Pathology Deaprtment, Tunis, Tunisia

\subsection{6/ijgc-2021-IGCS.214}

Objectives Endometrial cancer is the second gynecologic cancer. The varying tumors profile from country to country and the difference in the means available in each country have raised the need for a country-specific guideline. We aim to Present the Tunisian guideline for endometrial cancer

Methods All relevant international and national scientific literature available from 2016 to 2021 was used to establish this guideline.

Results This guideline was made by the Gynecologic Oncology Multidisciplinary team of the National Cancer center. Three questions were asked. What is the actual state of the art? Could it be applied in our country? If not, can we adapt the guideline to our reality?. During the consensus, the panel tried to cope between the actual state of the art and the Tunisian Field reality. The main limitations were the Distant radiation appointment, the patient loss to follow up, and the non-systematic use of biological markers. The 2009 FIGO classification was used to stage our patients. For stage I disease, The ESMO 2016 risk classification was used. One preoperatory and composed of three levels of risks low, intermediate, and high risk. The other classification is post-operatory and comprises low, Intermediate, high-intermediate, and high-risk levels. Based on this Data and our country reality panel developed recommendations.

Conclusions A country-specific guideline based on the international state of the art is more effective to offer the best quality of care available to our patients. It would also point to the lack and what needs to be done to keep on improving the health system.

\section{EPV145/\#82 MULTICENTRIC PREDICTIVE SCORE VALIDATION FOR NODAL ASSESSMENT IN ENDOMETRIAL CANCER PATIENTS: PRELIMINARY DATA}

${ }^{1}$ VA Capozzi, ${ }^{2} \mathrm{G}$ Sozzi, ${ }^{3} \mathrm{~A}$ Rosati ${ }^{*},{ }^{3} \mathrm{~S}$ Restaino, ${ }^{1} \mathrm{G}$ Gambino, ${ }^{1} \mathrm{~A}$ Cianciolo, ${ }^{4} \mathrm{M}$ Ceccaroni, ${ }^{2} \mathrm{~V}$ Chiantera, ${ }^{5} \mathrm{~S}$ Uccella, ${ }^{5} \mathrm{M}$ Franchi, ${ }^{3} \mathrm{G}$ Scambia, ${ }^{3} \mathrm{~F}$ Fanfani, ${ }^{1} \mathrm{R}$ Berretta. ${ }^{1}$ University of Parma, Department of Medicine and Surgery, Parma, Italy; ${ }^{2}$ University of Palermo, Department of Gynecologic Oncology, Arnas Civico Di Cristina Benfratelli, Palermo, Italy; ${ }^{3}$ Università Cattolica del Sacro Cuore, Department of Woman and Child Health and Public Health, Woman Health Area, Fondazione Policlinico Universitario A. Gemelli Irccs, Roma, Italy; ${ }^{4}$ RCCS Sacro Cuore Don Calabria Hospital, Gynecologic Oncology, Minimally-Invasive Pelvic Surgery, International School of Surgical Anatomy, Negrar, Italy; ${ }^{5}$ University of Verona, Department of Obstetrics and Gynecology, Verona, Italy

\subsection{6/ijgc-2021-IGCS.215}

Objectives Sentinel lymph node (SLN) is considered the standard of care in early-stage endometrial cancer (EC) patients. In case of SLN failure, a side-specific lymphadenectomy of the 
no mapping hemipelvis is recommended. Nevertheless, most hemipelvis lymphadenectomies showed no nodal involvement. Previously, we published a preoperative predictive score of nodal involvement. In case of a negative score (value 3-4), the risk of nodal metastases was extremely low. The present multicentre study aims to validate the predictive score of nodal involvement in patients undergoing nodal assessment.

Methods EC patients undergoing surgical treatment with nodal staging were included in the analysis. A preoperative predictive score of nodal involvement was calculated for all patients before surgery was performed. The score included myometrial infiltration, tumor grading $(\mathrm{G})$, tumor diameter, and Ca125 assessment. STARD (standards for Reporting Diagnostic accuracy studies) guidelines were followed for the score accuracy. Results 1038 patients were included in the analysis and 155 (14.9\%) nodal metastases were detected. The score was negative ( 3 and 4) in 475 patients and positive (5-7) in 563 cases. The score showed $83.2 \%$ sensitivity, $50.8 \%$ specificity, $94.5 \%$ negative predictive value, and $55.7 \%$ diagnostic accuracy. The area under the curve (AUC) was 0.75. The logistic regression between negative score and absent nodal metastases showed OR 5.133, 95\% CI (3.30-7.98), p <0.001.

Conclusions The nodal preoperative predictive score is a fair diagnostic test. The risk of nodal metastasis is extremely low in case of negative score. In SLN failure, the application of the present score associated with SLN algorithm could avoid unnecessary lymphadenectomies.

\section{EPV146/\#133 ACCEPTABILITY OF BARIATRIC SURGERY IN YOUNG WOMEN WITH ENDOMETRIAL CANCER AND ATYPICAL ENDOMETRIAL HYPERPLASIA: A QUALITATIVE STUDY}

${ }^{1}$ RS Kim*, ${ }^{1} \mathrm{G}$ Ene, ${ }^{2} \mathrm{~A}$ Simpson, ${ }^{3} \mathrm{D}$ Gesink, ${ }^{1} \mathrm{~S}$ Ferguson. ${ }^{1}$ Princess Margaret Cancer Centre/ University of Health Network/Sinai Health Systems, Gynecologic Oncology, Toronto, Canada; ${ }^{2}$ St.Michael's Hospital/Unity Health Toronto, Obstetrics and Gynecology, Toronto, Canada; ${ }^{3}$ University of Toronto, Dalla Lana School of Public Health, Toronto, Canada

\subsection{6/ijgc-2021-IGCS.216}

Objectives Endometrial cancer (EC) or atypical hyperplasia $(\mathrm{AH})$ in young women with obesity is often the first significant obesity-related comorbidity they experience. Significant, sustained weight loss through bariatric surgery may result in a durable response by addressing obesity directly, and subsequently improve oncologic and reproductive outcomes. However, it is not known whether bariatric surgery is acceptable to this patient population.

Methods We performed a qualitative study to understand the acceptability of bariatric surgery among women of reproductive age with $\mathrm{BMI} \geq 35$ and grade $1 \mathrm{EC} / \mathrm{AH}$. Semi-structured interviews were used to explore participant perceptions towards their weight, fertility, and the possibility of bariatric surgery as part of the treatment strategy for their EC/AH.

Results Eleven participants with median age of 33 years (range 27-38) and BMI of 42.1 (35.1-56.9) were interviewed. Two (18\%) participants had grade $1 \mathrm{EC}$, and 9 (82\%) had $\mathrm{AH}$. Patients were reluctant to accept bariatric surgery as a treatment option due to 1) lack of knowledge about the procedure, 2) stigma attached to bariatric surgery, and 3) fear of the unknown. The desire to conceive was highlighted as the strongest motivator for patients to consider bariatric surgery. Their perception towards their weight, fertility and diagnosis of EC/AH were characterized by concepts of 'helplessness', 'isolation', 'frustration' and 'guilt'. We observed a significant gap in participant understanding of the complex interplay between their cancer, fertility and obesity.

Conclusions We need to provide patient-oriented counseling on implication of their weight on their cancer and fertility, before presenting bariatric surgery as a treatment option.

\section{EPV147/\#253 LIVE BIRTH, REMISSION AND RELAPSE RATES FOR FERTILITY-PRESERVING TREATMENTS OF ENDOMETRIAL ADENOCARCINOMA: A SYSTEMATIC REVIEW AND META-ANALYSIS}

S Oxley*, O Ogunbiyi, R Graham, A Olaitan. UCLH, Women's Health, London, UK

\subsection{6/ijgc-2021-IGCS.217}

Objectives Endometrial adenocarcinoma affects over 380,000 women annually, with increasing incidence primarily driven by obesity. 5-7\% of women are below 45 years at diagnosis, and many of these desire fertility-preservation rather than standard surgical treatment. This updated review aims to inform decision making in clinical practice, by evaluating the efficacies of different fertility-preserving treatments on the live birth, regression and relapse rates for women with endometrial carcinoma desiring fertility.

Methods A systematic search was performed of Medline, Embase, Central, \& Cochrane, to identify studies describing fertility-preserving treatment for endometrial cancer. Patients were divided into 3 treatment groups: systemic progestogens, intra-uterine progestogens, or hysteroscopic resection with adjuvant progestogen. A random-effects meta-analysis model was used.

Results 41 observational studies met inclusion criteria, with 1057 patients in total. The proportion of women receiving systemic progestogens who achieved a live birth was $18.1 \%$ (95\% CI 12.6-23.7\%), remission $71.5 \%$ (95\% CI $66.5-$ $76.4 \%$ ) and relapse $20.3 \%$ (95\% CI 13.1-27.4\%). For intrauterine progestogens, the proportion achieving a live birth was $13.3 \% \quad(95 \%$ CI $11.1-15.5 \%)$, remission $65.9 \% \quad(95 \%$ CI $53.0-78.8 \%$ ) and relapse $2.86 \%$ (95\% CI 0.0-9.16\%). For hysteroscopic resection, the proportion achieving a live birth was $19.1 \%$ (95\% CI $8.79-29.5 \%$ ), remission $82.7 \%$ (95\% CI 73.1-92.3\%) and relapse $6.80 \%$ (95\% CI 1.72-11.9\%).

Conclusions Although the quality of evidence is limited, these results demonstrate that hysteroscopic resection with adjuvant progestogen is associated with the highest rates of live birth and remission. This enables women considering such treatments to be fully counselled on the realistic possibilities of their desired reproductive and oncological outcomes.

\section{EPV148/\#91 OUTCOMES OF VARIOUS FERTILITY-SPARING OPTIONS FOR EARLY CERVICAL CANCER PATIENTS VERSUS ABDOMINAL RADICAL HYSTERECTOMY: ONE CANCER CENTER TEN-YEAR EXPERIENCE}

O Matylevich*, V Petukhou, I Bakinouskaya, S Taranenka. NN Alexandrov National Cancer Centre of Belarus, Gynecologic Oncology Department, Minsk, Belarus

\subsection{6/ijgc-2021-IGCS.218}

Objectives Cervical cancer (CC) is one of the most common malignant neoplasms and is diagnosed at the youngest middle 